\title{
Kinematics of roller chain drives - Exact and approximate analysis
}

\author{
Fuglede, Niels; Thomsen, Jon Juel
}

Published in:

Mechanism and Machine Theory

Link to article, DOI:

10.1016/j.mechmachtheory.2016.01.009

Publication date:

2016

Document Version

Peer reviewed version

Link back to DTU Orbit

Citation (APA):

Fuglede, N., \& Thomsen, J. J. (2016). Kinematics of roller chain drives - Exact and approximate analysis. Mechanism and Machine Theory, 100, 17-32. https://doi.org/10.1016/j.mechmachtheory.2016.01.009

\section{General rights}

Copyright and moral rights for the publications made accessible in the public portal are retained by the authors and/or other copyright owners and it is a condition of accessing publications that users recognise and abide by the legal requirements associated with these rights.

- Users may download and print one copy of any publication from the public portal for the purpose of private study or research.

- You may not further distribute the material or use it for any profit-making activity or commercial gain

- You may freely distribute the URL identifying the publication in the public portal

If you believe that this document breaches copyright please contact us providing details, and we will remove access to the work immediately and investigate your claim 


\title{
Kinematics of Roller Chain Drives - Exact and Approximate Analysis
}

\author{
Niels Fuglede, Jon Juel Thomsen ${ }^{1}$, \\ Department of Mechanical Engineering, Technical University of Denmark, Building 404, \\ DK-2800, Lyngby, Denmark
}

\begin{abstract}
An exact and approximate kinematic analysis of a roller chain drive modeled as a four-bar mechanism is presented. The span connects the sprockets such that they rotate in the same direction, and the sprocket size, number of teeth, and shaft center distance can be arbitrary. The driven sprocket angular position, velocity and acceleration, as well as span length, is calculated and their (discontinuous) variation with driver angular position and main design parameters is illustrated. Kinematic predictions for the chain span motion are compared to results of multibody simulation, and there is seen to be very good agreement. All together this gives new insights into the characteristics of chain drive kinematics and the influence of main design parameters.
\end{abstract}

Keywords: Roller chain, chain drives, kinematics, four-bar mechanism, multibody simulation

\footnotetext{
${ }^{1}$ Corresponding author: jjt@mek.dtu.dk
} 


\section{Introduction}

Roller chain drives are widely used machine elements due to high energy efficiency and timing capabilities. Research topics include kinematics, chain span dynamics, load distributions, coupled sprocket and span dynamics, alternative design, multibody dynamics, roller impact and noise emission. A literature survey of noise and vibration was given by Wang and Liu [1]. Belt drives is a related research area and a survey including also this was given by Fawcett [2]. Also relevant for chain span dynamics are the studies of axially moving materials, surveyed by Chen [3].

The discrete nature of a chains introduce several effects, collectively known as polygonal action. Some of these effects are less desirable, e.g. the uneven transfer of torques between the sprockets and impact between chain rollers and sprockets. Polygonal action is inherent of chain drives, because a chain wrapped around sprockets form polygons rather than circles.

In chain drive kinematics, mass and elasticity are neglected and usually also tolerances and manufacturing inaccuracies of the drive components. Results therefore describe the motion of ideal chain drives operating at nonresonant conditions and very low speeds. The main object of investigation is often the velocity of the driven sprocket, and parameters of main concern are shaft center distance and tooth ratio. A detailed kinematic analysis reveals the characteristic loading of the chain drive and may aid the interpretation of simulation- and measurement results.

Early studies of the kinematics includes the work by Bartlett [4], who observed that sprockets could be modeled as polygons. He derived an expression for the minimum and maximum variation of the angular velocity ratio. He also noted that these are obtained when sprockets are positioned 
such that the length of the driving span equals an integer number of pitch lengths, or an odd number of half pitches, respectively. In the work by Morrison [5] the kinematic motion of the chain drive is recognized to happen through a series of four-bar mechanisms. An expression for the shaft center distance giving the smallest velocity ratio variation was given. It was then shown how shaft center distance influence the angular acceleration of the driven sprocket, and thereby the chain drive loads.

A full monograph was written by Binder [6] on roller chain drives containing treatments of many subjects relevant for chain drive designers, including standard tooth geometry, static loading, velocity variations as well as friction and wear. The dynamics of the driven sprocket was considered by Mahalingam [7], who expressed the tension variation of the chain span due to polygonal action using the first harmonic term of a Fourier approximation. With this approximation, the driven sprocket is subjected to monofrequency forced vibration and high-frequency components originating from impact loading and discontinuity are unaccounted for. The periodic fluctuations of driven sprocket velocity was studied by Bouillon and Tordion [8] both numerically and experimentally. An approximate analysis, also of the driven sprocket velocity, was made by Turnbull and Fawcett [9], who expressed the driven sprocket velocity as a series expansion, and illustrated the influence of the number of expansion terms for different centre distances and tooth ratios. A general kinematic analysis was presented by Chen and Freudenstein [10], where the shaft center distance could be arbitrary. The configuration of the chain drive where a roller seats on the driver sprocket was determined. The kinematic analysis also highlighted the discontinuous variation of span length, angular velocity- and acceleration ratios. Standards for design and dimensions of roller chains and sprockets are maintained by 
organizations such as ANSI, BS and DIN.

Numerical analysis of roller chain drive systems has been developed using a multibody modeling approach by Pedersen et al. [11], which can take into account non-linear dynamic coupling, exact tooth geometry and impact phenonema [12]. A general methodology for planar models of multibody chain drives has been suggested by Pereira et al. [13]. Models that can include joint clearances are presented by [14].

In this study we present an exact kinematic analysis of the motion of the chain span components, i.e. the position of the span endpoints as well as angular position, velocity and acceleration of the driven sprocket during one tooth-period of the driver sprocket. Approximate results for the motion of the driven sprocket are also derived, based on the exact results. Simple approximate expressions for the seating and release configurations are derived, and these are used for obtaining the first analytical expression for the phase between rollers seating and releasing. The exact and approximate results are compared and shown to be in very good agreement for practical chain drives. The approximate results significantly ease the calculation of the motion of the driven sprocket, and may aid designers to quickly evaluate designs and estimate chain drive loads. The obtained results include the discontinuous properties of the driven sprocket motion, as opposed to the existing approximate analysis [9]. Comparison is made between kinematic predictions of the chain span path, and results multibody simulation. There is seen to be very good agreement and the kinematic analysis proves useful for interpreting the simulation results. 


\section{Exact kinematic analysis}

The purpose of the analysis presentation is to make a clear illustration and derivation of the kinematic movement of the chain drive modeled as a four-bar mechanism. This presentation reworks and expands on results presented [10], but with motion coordinates better suited for approximate analysis. Attention is on presenting a self contained analysis, with complete results and clear derivations.

\subsection{Kinematic model}

The kinematic model defines how the chain drive elements and their dimensions are simplified as rigid components connected by perfect frictionless joints. The assumptions, geometry, coordinate system for the model, and the governing equations are presented in the following.

\subsection{Assumptions}

In the kinematic analysis the geometry of the chain and sprockets are assumed to be a perfect match, i.e. 1) sprocket pitch is equal to chain pitch, 2) the chain drive is without any mechanical clearances, and 3) a roller seated on the sprocket is positioned in the center of the sprocket seating curve. Neglecting dynamic effects introduce the following assumptions: 4) the span is perfectly straight, 5) chain and sprocket elements are rigid, and 6) the system is frictionless.

These assumptions neglect some features of real chain drives, e.g. the detailed tooth geometry, clearances, imperfect geometry, as well as chain imperfections. These are considered of minor importance for predicting the main aspects of the kinematic characteristics, which can be derived from shaft center distance, number of teeth and pitch length. Including 
geometric details or imperfections would complicate or (render impossible) analytical approximations that are simple enough to provide insight. Some of the assumptions are relaxed in the numerical analysis used for validating analytical approximations, e.g. standard tooth geometry is implemented, so that their effects can be directly assessed.

\subsection{Basic geometry}

Consider the sketch of a chain meshing with at sprocket in Fig. 1. Rollers are shown as small circles, thereby marking the chain as a connection of rigid links. The chain moves from left to right and the roller $S_{N}$ will be removed from the free span as the sprocket rotates clockwise around point $C$. Shown in the sketch is the exact moment where roller $B_{1}$ gets in contact with the sprocket and thereby define the new endpoint for the span.

Several geometric properties can be defined from Fig. 1. The pitch, $p$, is the distance between two chain rollers and also the length of the sides of the pitch polygon, witch is formed by connecting the centers of the sprocket seating curves. Half the angle between two seating curve centers is referred to as the pitch angle $\alpha$. The pitch polygon has inscribed circle radius $r$ and the circle drawn out by the centers of the seating curves on the sprockets is referred to as the pitch circle and has radius $R$. By formula, these variables are given by, respectively,

$$
\alpha=\frac{\pi}{m}, \quad r=\frac{p}{2 \tan \alpha}, \quad R=\frac{p}{2 \sin \alpha},
$$

where $m$ is the number of teeth on the sprocket.

It is seen from Fig. 1 that the length and endpoint positions of the free span varies discontinuously as the sprocket rotate clockwise around $C$. Rotation of the sprocket causes a vertical movement of the span endpoint 
between values $r$ and $R$, and a horizontal movement between positions $B_{1}$ and $B_{2}$. The angle between the chain span $S_{N} B_{1}$ and the line $C B_{1}$ varies during rotation of the sprocket, and therefore a constant driving torque will not be transmitted evenly to the chain. Vectors $\mathbf{u}_{1}$ and $\mathbf{u}_{2}$ indicate the sprocket velocities at rollers $B_{1}$ and $B_{2}$, respectively. These velocities differ in direction and this cause an impact between the roller $B_{1}$ and the sprocket.

Collectively, these effects are referred to as polygonal action, their magnitude decrease as the number of sprocket teeth increase, but remains finite and, a characteristic for roller chain drives.

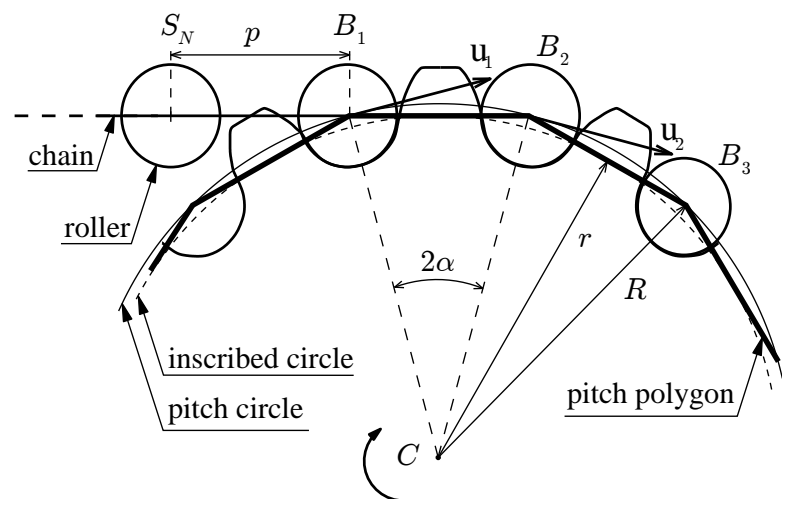

Figure 1: Sprocket meshing with chain

\subsection{Coordinate system}

Figure 2 shows the kinematic model in the coordinate system used throughout the analysis. The driving sprocket constraining the motion is centered at $C$ and the driven sprocket at $O$. Subscripts $c$ and $o$ will be used to refer to those sprockets, e.g. $\alpha_{o}$ and $R_{c}$. Both sprockets are drawn as pitch polygons, connected by a line $A_{1} B_{1}$ representing the tight chain span. The main object of investigation is the tight span, since it transfers the torque from the driving sprocket to the driven sprocket. 
When the chain drive consists of only two sprockets there will be a slack chain span connecting the two sprockets opposite of the tight span, and this will not be considered in the analysis. In case there are more than two sprockets there will be no slack span between sprockets $O$ and $C$ and the analysis presented here covers the transfer of torque between two neighboring sprockets in a larger chain drive system.

The origin of the fixed Cartesian $X Y$-coordinate system is coincident with the center $O$ of the driven sprocket. It is orientated such that the $X$ axis is parallel with the tangent $T_{o} T_{c}$ common to the two inscribed circles, so that the coordinates $\left(x_{c}, y_{c}\right)$ of $C$ is:

$$
x_{c}=\left|T_{o} T_{c}\right|, \quad y_{c}=r_{o}-r_{c} .
$$

For a wide range of typical chain drives lines $O A_{1}$ and $C B_{1}$, make small variations around the vertical direction and the slope of the span make small variations around the horizontal direction, while $O C$ remain fixed. The smallness in variations makes the kinematic model suitable for approximate analysis.

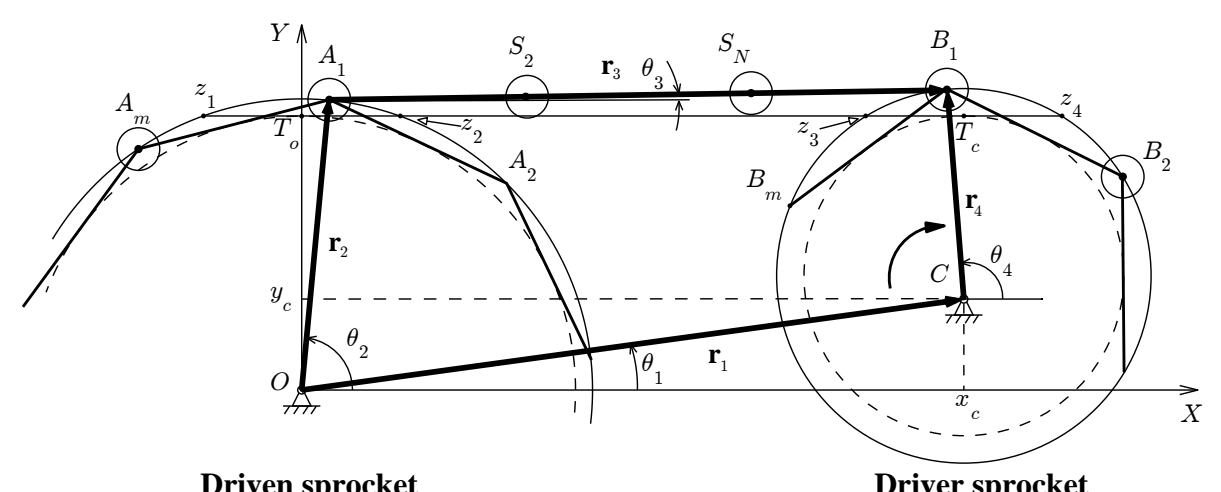

Figure 2: Chain drive kinematically modeled as a four-bar mechanism 


\subsection{Span length}

When the roller chain drive is in operation, chain links will recurrently enter and leave the chain span and consequently the span length varies discontinuously with sprocket angular rotation, c.f. Fig. 2. The driving sprocket constrain the motion, and as it rotates the angle $2 \alpha_{c}$ in clockwise direction, one tooth period passes and two events occurs: Roller $A_{1}$ loses contact with the driven sprocket and is released into the free span. It happens at the instant where the slope of $A_{m} A_{1}$ equals the slope of the span, and shifts the span endpoint from roller $A_{1}$ to $A_{m}$, thereby increasing span length by one pitch. Similarly, roller $S_{N}$ gets in contact with the driving sprocket at the instant where the slope of $B_{m} B_{1}$ equals the slope of the span. This makes $S_{N}$ the new span endpoint, thereby decreasing the length of the span by one pitch.

The length of the span and its dependency on design parameters is determined next. In Fig. 2, the common inner tangent $T_{o} T_{c}$ of the inscribed circles intersect the pitch circle of sprocket $O$ in points $z_{1}$ and $z_{2}$. Similarly, the tangent intersects the pitch circle of sprocket $C$ in points $z_{3}$ and $z_{4}$. The length $\left|z_{1} z_{2}\right|$ equals one pitch, as do $\left|z_{3} z_{4}\right|$. The span $A_{1} B_{1}$ must equal an integral number of pitches and its length can be determined using the points $z_{1}, z_{2}, z_{3}$, and $z_{4}$. From Fig. 2 one finds that the chain span length $\left|A_{1} B_{1}\right|$ fulfills

$$
\left|z_{2} z_{3}\right| \leq\left|A_{1} B_{1}\right| \leq\left|z_{1} z_{4}\right|
$$

where $\left|z_{2} z_{3}\right|=\left|T_{o} T_{c}\right|-p$ and $\left|z_{1} z_{4}\right|=\left|T_{o} T_{c}\right|+p$. Generally $\left|z_{2} z_{3}\right|$ equals an integer number of pitches $N$ plus a fraction of pitch lengths $f$, both defined from

$$
\left|z_{2} z_{3}\right|=(N+f) p, \quad f \in[0,1[, \quad N \in \mathbb{N} .
$$


In the chosen coordinate system the length of the common inner tangent $\left|T_{o} T_{c}\right|$ equals $x_{c}$; using (4) its length can be expressed as

$$
x_{c}=\left|T_{o} T_{c}\right|=(N+1+f) p .
$$

With this (3) can be written

$$
(N+f) p \leq\left|A_{1} B_{1}\right| \leq(N+2+f) p .
$$

In the general case where $f \neq 0$ the span length $\left|A_{1} B_{1}\right|$ fulfills the inequality (6) with two solutions,

$$
\left|A_{1} B_{1}\right|=(N+j) p, j=1,2, \text { for } f \neq 0 .
$$

The span length varies discontinuously between these two values when a roller is seated on the driver sprocket, and released from the driven sprocket, respectively.

In the special case where $f=0$ the common inner tangent equals an integral number of pitches. Then inequality (6) has three solutions for the span length: $\left|A_{1} B_{1}\right|=(N+j) p, j=0,1,2$, for $f=0$. However, these solutions exist simultaneously, and only in one instant, which is the specific configuration of the chain drive where $A_{m} A_{1} B_{m} B_{1}$ align and coincide with points $z_{1} z_{2} z_{3} z_{4}$, respectively, c.f. Fig. 2. Further rotation of the sprockets from this configuration will cause roller $A_{1}$ to be released from the driven sprocket, making $A_{n}$ the new span endpoint. At the same time, roller $S_{N}$ is seated on the driver sprocket, making $B_{m}$ the new span endpoint. Thus, when $f=0$, the release and seating of rollers happens simultaneously and the span length remains constant at

$$
\left|A_{1} B_{1}\right|=(N+1) p, \text { for } f=0 .
$$


With the above it has been determined how the chain span length generally shifts between two values, depending on the design parameters, i.e. the common inner tangent length $\left|T_{o} T_{c}\right|$, pitch length $p$, and pitch fraction $f$. How the length varies depends on the driver position, as will be determined in the following.

\subsection{Governing vector equations}

The vector equations presented here describe the position-, velocity- and acceleration relations for the kinematic model of the chain drive. For ease of notation, the vectors in the four-bar mechanism in Fig. 2 are written in polar form as $\mathbf{r}_{n}=r_{n} e^{i \theta_{n}}, n=1,2,3,4$, where $r_{n}$ is the length of the vector, $\theta_{n}$ the orientation measured positive counter clockwise from the $X$-axis, and the real and imaginary parts of the vector are parallel to the $X$ - and $Y$-axis, respectively. The vectors in the four-bar mechanism are defined as

$$
\mathbf{r}_{1}=O C, \quad \mathbf{r}_{2}=O A_{1}, \quad \mathbf{r}_{3}=A_{1} B_{1}, \quad \mathbf{r}_{4}=C B_{1}
$$

Sprocket centers do not move and therefore $\mathbf{r}_{1}$ is constant with time. From Fig. 2, the equation governing the position of link joint $B_{1}$ is

$$
\mathbf{r}_{2}+\mathbf{r}_{3}=\mathbf{r}_{1}+\mathbf{r}_{4}
$$

sometimes referred to as the closure- or four-bar equation. For $n=2,3,4$, angular velocities and accelerations are introduced as $\omega_{n}=d \theta_{n} / d t$ and $\alpha_{n}=$ $d \omega_{n} / d t$, respectively. Differentiating (10) with respect to time gives, since $d v_{n} / d t=0$, the equation relating the velocities

$$
i \omega_{2} \mathbf{r}_{2}+i \omega_{3} \mathbf{r}_{3}-i \omega_{4} \mathbf{r}_{4}=\mathbf{0}
$$


Finally, differentiating (11) with respect to time gives the equation for the accelerations

$$
\left(i \alpha_{2}-\omega_{2}^{2}\right) \mathbf{r}_{2}+\left(i \alpha_{3}-\omega_{3}^{2}\right) \mathbf{r}_{3}-\left(i \alpha_{4}-\omega_{4}^{2}\right) \mathbf{r}_{4}=\mathbf{0}
$$

The factor $i=\sqrt{-1}$ in the coefficient of a vector indicates a direction perpendicular to that vector, taken in clockwise direction. Equations (11) and (12) represent the velocity and acceleration vector diagrams, respectively [15].

As noted above, the span length shifts between two fixed values as rollers are seated and released from the sprockets. It is useful to express span length $r_{3}=\left|A_{1} B_{1}\right|$ using the lower value of the span length, introduced here as $L$, and a step function $h$. The function $h$ is unity when the span length assumes its upper value and zero otherwise. Vectors $\mathbf{r}_{2}$ and $\mathbf{r}_{4}$ have constant length given by pitch circle radii and, $r_{1}=|O C|$ can be determined from Fig. 2, thus

$$
r_{1}=\sqrt{x_{c}^{2}+y_{c}^{2}}, r_{2}=R_{o}, r_{3}=L+h p, r_{4}=R_{c}, L \equiv(N+1) p
$$

A formal definition of the function $h=h\left(\theta_{4}\right)$ is given below using the seating and release configurations.

\subsection{Introduction to position analysis}

The configurations of the chain drive for which a roller is just seated on the driver sprocket and just released from the driven sprocket, are determined first. Between these two events, the span length remain constant. The angular position of the driven sprocket during the rotation of the driver sprocket is then determined in the subsequent input-output analysis.

Splitting (10) into real and imaginary parts gives the equations to be 
solved in order to determine the positions of the four-bar members:

$$
\begin{aligned}
& r_{2} \cos \theta_{2}+r_{3} \cos \theta_{3}=r_{1} \cos \theta_{1}+r_{4} \cos \theta_{4} \\
& r_{2} \sin \theta_{2}+r_{3} \sin \theta_{3}=r_{1} \sin \theta_{1}+r_{4} \sin \theta_{4},
\end{aligned}
$$

which can also be derived directly from Fig. 2 .

\subsection{Configuration with roller just seated on driver sprocket}

Figure 3(a) shows the four-bar mechanism $O A_{1} B_{1} C$ in the exact configuration where roller $B_{1}$ seats on the driver sprocket $C$, making $B_{1}$ the new span endpoint. Figure 3(b) shows the configuration when a roller is just released from the driven sprocket. Variables related to the seating and release of a roller are subscripted $s$ and $r$, respectively.

When determining the seating and release configurations, the span length in both cases attain its lower value. Therefore all lengths $r_{n}$ are given by (13) with $h=0$. What then remains for the configuration to be determined are the angles $\theta_{n}, n=2,3,4$. At point $B_{1}$ in Fig. $3(\mathrm{a})$ it is seen that

$$
\theta_{3 s}=\theta_{4 s}-\gamma,
$$

where $\gamma=\pi / 2+\alpha_{c}$ is known, cf. (1). When $\theta_{3 s}$ is expressed in terms of $\theta_{4 s}$ and $\gamma$, there are two remaining unknowns to be determined from (14)-(15), namely $\theta_{2 s}$ and $\theta_{4 s}$. To do this one can first square and add (14) and (15) to eliminate $\theta_{2 s}$. Expanding the squared terms, and also the trigonometric terms containing $\theta_{3 s}$ using (16), e.g., $\sin \theta_{3 s}=\sin \theta_{4 s} \cos \gamma-\cos \theta_{4 s} \sin \gamma$, gives

$$
A_{s} \cos \theta_{4 s}+B_{s} \sin \theta_{4 s}+C_{s}=0,
$$


(a)

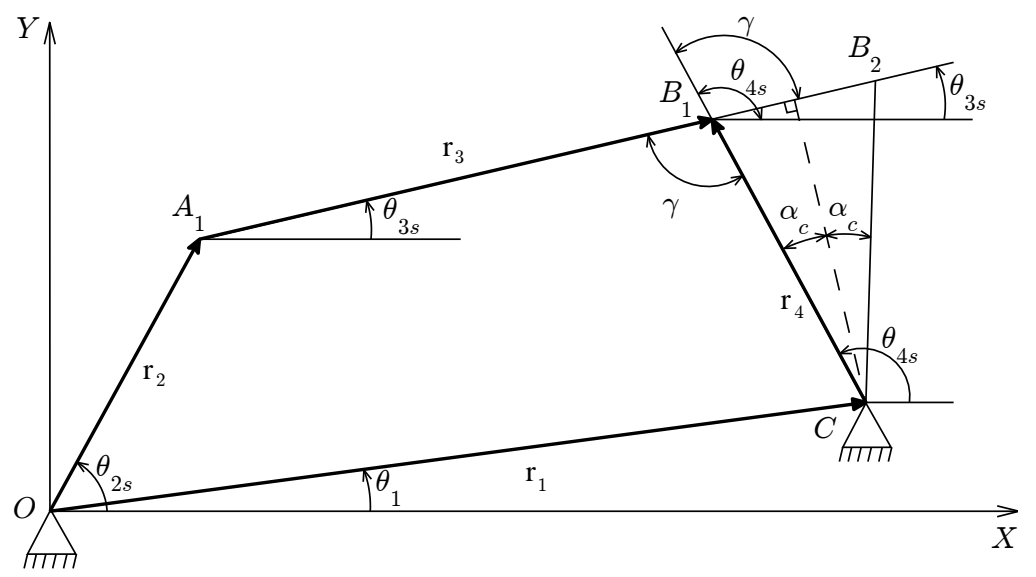

(b)

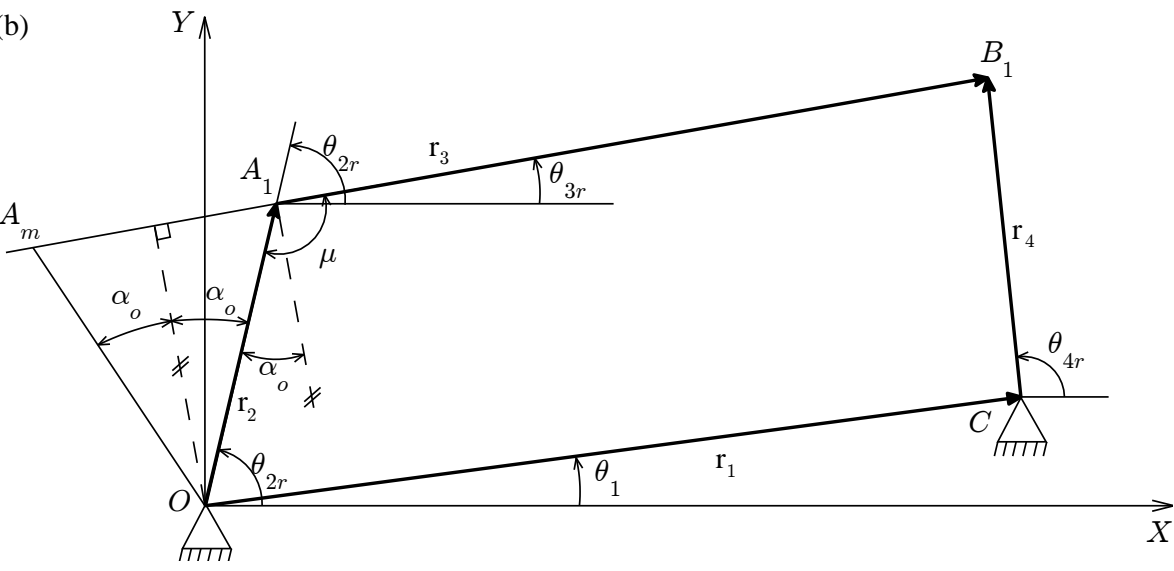

Figure 3: Equivalent four-bar mechanism when a roller (a) just seats on sprocket $C$, and (b) just releases from sprocket $O$. 
with coefficents

$$
\begin{aligned}
& A_{s}=2 r_{1} r_{4} \cos \theta_{1}-2 r_{1} r_{3}\left(\cos \theta_{1} \cos \gamma-\sin \theta_{1} \sin \gamma\right), \\
& B_{s}=2 r_{1} r_{4} \sin \theta_{1}-2 r_{1} r_{3}\left(\cos \theta_{1} \sin \gamma+\sin \theta_{1} \cos \gamma\right), \\
& C_{s}=r_{1}^{2}+r_{4}^{2}+r_{3}^{2}-r_{2}^{2}-2 r_{4} r_{3} \cos \gamma .
\end{aligned}
$$

Equations in the form of (17)-(18) will reappear in the following sections and it is therefore useful to present the solution in a general formulation. Consider the equation $A \cos \theta+B \sin \theta+C=0$, from which $\theta$ is to be determined [16]. By introducing $\sin \theta=2 \tau /\left(1+\tau^{2}\right), \cos \theta=\left(1-\tau^{2}\right) /\left(1+\tau^{2}\right)$, with $\tau=\tan (\theta / 2)$, the equation can be written as a second order polynomial in $\tau$, with solutions

$$
\tau_{1,2}=\frac{-B \pm \sqrt{B^{2}-C^{2}+A^{2}}}{C-A} .
$$

The two solutions for $\theta$ are then

$$
\theta=2 \arctan \left(\tau_{1,2}\right)
$$

Following this approach, the solution for $\theta_{4 s}$ is given by (20) when the coefficients in (18) are substituted into (19). With $\theta_{4 s}$ determined, the solution for $\theta_{3 s}$ is given by (16). Finally, $\theta_{2 s}$ is determined by division of (15) with (14), leading to

$$
\theta_{2 s}=\arctan \left(\frac{r_{1} \sin \theta_{1}+r_{4} \sin \theta_{4 s}-r_{3} \sin \theta_{3 s}}{r_{1} \cos \theta_{1}+r_{4} \cos \theta_{4 s}-r_{3} \cos \theta_{3 s}}\right) .
$$

There are two solutions sets, corresponding to the two values of $\tau$ in (19). These correspond to configurations where the sprockets are connected by the span to rotate either in the same or opposite directions. The two solutions for $\theta_{2 s}$ are of opposite sign, and the solution-set where $\theta_{2 s}>0$ is the one for which both sprockets rotate in the same direction. 


\subsection{Configuration with roller just released from the driven sprocket}

Figure $3(\mathrm{~b})$ shows the configuration where roller $A_{1}$ loses contact with the driven sprocket $O$ and thereby enters the span, making $A_{n}$ the new span endpoint. At this event $\theta_{3}$ attains the value

$$
\theta_{3 r}=\mu+\theta_{2 r}-\pi
$$

where $\mu=\pi / 2+\alpha_{o}$, the span length is at its lower value, and all lengths are given by (13) with $h=0$. With $\theta_{1}$ known and fixed, and $\theta_{3 r}$ given by (22), $\theta_{2 r}$ and $\theta_{4 r}$ remain to be determined from (14)-(15). We do this by squaring and adding (14) and (15) to eliminate $\theta_{4 r}$, expanding the squared terms, substituting (22) for $\theta_{3 r}$, and expanding using trigonometric relations, e.g. $\cos \theta_{3 r}=\cos \theta_{2 r} \cos (\mu-\pi)-\sin \theta_{2 r} \sin (\mu-\pi)$, and finds:

$$
A_{r} \cos \theta_{2 r}+B_{r} \sin \theta_{2 r}+C_{r}=0
$$

with coefficients,

$$
\begin{aligned}
& A_{r}=-2 r_{2} r_{1} \cos \theta_{1}-2 r_{3} r_{1}\left(\cos \theta_{1} \cos (\mu-\pi)+\sin \theta_{1} \sin (\mu-\pi)\right), \\
& B_{r}=-2 r_{2} r_{1} \sin \theta_{1}+2 r_{3} r_{1}\left(\cos \theta_{1} \sin (\mu-\pi)-\sin \theta_{1} \cos (\mu-\pi)\right), \\
& C_{r}=r_{2}^{2}+r_{3}^{2}+r_{1}^{2}-r_{4}^{2}+2 r_{2} r_{3} \cos (\mu-\pi) .
\end{aligned}
$$

Substituting these coefficients into (19), the solution for $\theta_{2 r}$ is given by (20). The angle $\theta_{3 r}$ is then found from (22), and $\theta_{4 r}$ from (14) and (15):

$$
\theta_{4 r}=\arctan \left(\frac{r_{2} \sin \theta_{2 r}+r_{3} \sin \theta_{3 r}-r_{1} \sin \theta_{1}}{r_{2} \cos \theta_{2 r}+r_{3} \cos \theta_{3 r}-r_{1} \cos \theta_{1}}\right) .
$$

As for $\theta_{2 s}$ the solution for which $\theta_{4 r}>0$ correspond to the configuration where the sprockets rotate in the same direction. 


\subsection{Span length variation}

Calculation of the driven sprocket angular position, velocity and acceleration using both exact and approximate methods can now take into account that the span length $r_{3}$ varies according to (13), with the function $h$ defined as:

$$
h=h\left(\theta_{4}\right)=\left\{\begin{array}{l}
0 \text { for } \theta_{4 s} \geq \theta_{4} \geq \theta_{4 r} \\
1 \text { for } \theta_{4 r} \geq \theta_{4} \geq \theta_{4 s}+2 \alpha_{c}
\end{array}\right.
$$

where $\theta_{4}$ is decreasing since the driver rotates in clockwise direction. The angles $\theta_{4 s}$ and $\theta_{4 r}$ are cumbersome to determine exactly, but simple and accurate approximations can be determined (sections 3.1 and 3.2).

\subsection{Input-output angular position}

In order to determine the motion of the driven sprocket it is required that the angular position, velocity and acceleration of the driver sprocket are known, i.e. that $\theta_{4}, \omega_{4}$ and $\alpha_{4}$ are given functions of time.

Determining $\theta_{2}$ as a function of $\theta_{4}$ follows steps similar to the ones carried out in the above analysis for the seating and release configurations. By squaring and adding (14)-(15) to eliminate $\theta_{3}$, expanding the squared terms and canceling out terms, the equation governing the output position $\theta_{2}$ can be written in the form

$$
A \cos \theta_{2}+B \sin \theta_{2}+C=0
$$

where

$$
\begin{aligned}
& A=-2 r_{1} r_{2} \cos \theta_{1}-2 r_{4} r_{2} \cos \theta_{4}, \\
& B=-2 r_{1} r_{2} \sin \theta_{1}-2 r_{4} r_{2} \sin \theta_{4}, \\
& C=r_{1}^{2}+r_{4}^{2}+r_{2}^{2}-r_{3}^{2}+2 r_{1} r_{4}\left(\cos \theta_{1} \cos \theta_{4}+\sin \theta_{1} \sin \theta_{4}\right) .
\end{aligned}
$$


Using these coefficients the solution for $\theta_{2}$ is given by (20), with $\tau_{1,2}$ determined from (19). For completeness, the solution for $\theta_{3}$ is found from (14)-(15) to be

$$
\theta_{3}=\arctan \left(\frac{r_{1} \sin \theta_{1}+r_{4} \sin \theta_{4}-r_{2} \sin \theta_{2}}{r_{1} \cos \theta_{1}+r_{4} \cos \theta_{4}-r_{2} \cos \theta_{2}}\right) .
$$

There are agin two solution sets, corresponding to the two values of $\tau$, where the two solutions for $\theta_{2}$ are of opposite sign, and $\theta_{2}>0$ corresponds to the configuration where the sprockets rotate in the same direction. With the chain span assumed to be straight, motion of the chain drive is toothperiodic, i.e. the position of the four-bar members repeat when the driver angle advances by $2 \alpha_{c}$, the angle between two consecutive teeth. During one period a roller will have been released into, and another one removed, from the span.

\subsection{Angular velocity}

The angular velocity of the driven sprocket $\omega_{2}$ generally varies with time, even if the driver sprocket rotates at constant angular velocity $\omega_{4}$. This velocity variation can be determined from (11): We split (11) into real and imaginary parts, eliminate $\omega_{3}$ from the real part using the imaginary part, expand the products of trigonometric functions, and find

$$
\omega_{2}=\omega_{4} \frac{r_{4}}{r_{2}} \frac{\sin \left(\theta_{4}-\theta_{3}\right)}{\sin \left(\theta_{2}-\theta_{3}\right)} .
$$

When $\theta_{3}$ is neglected in the above expression, the result agrees with existing approximate result [7]. Note here, that the driver velocity may vary, i.e. $\omega_{4}=\omega_{4}(t)$. The angular velocity $\omega_{3}$ of the span can be determined following an approach similar as for the calculation of $\omega_{2}$. This gives

$$
\omega_{3}=\omega_{4} \frac{r_{4}}{r_{3}} \frac{\sin \left(\theta_{2}-\theta_{4}\right)}{\sin \left(\theta_{2}-\theta_{3}\right)} .
$$




\subsection{Angular acceleration}

Accelerations of the driven sprocket are relevant for calculating forces transmitted to the chain drive components and attached machinery. The angular acceleration $\alpha_{2}$ of the driven sprocket depends both on the driver sprocket angular acceleration $\alpha_{4}$ and the sprocket angular velocities $\omega_{2}$ and $\omega_{4}$. From the real part of (12) we eliminate $\alpha_{3}$ using the imaginary part, simplify using sum and products of trigonometric functions, and rewrite to get the result:

$$
\alpha_{2}=\alpha_{4} \frac{\omega_{2}}{\omega_{4}}+\frac{\omega_{4}^{2} r_{4} \cos \left(\theta_{4}-\theta_{3}\right)-\omega_{2}^{2} r_{2} \cos \left(\theta_{2}-\theta_{3}\right)-\omega_{3}^{2} r_{3}}{r_{2} \sin \left(\theta_{2}-\theta_{3}\right)},
$$

where the first term has been simplified using (30).

\section{Approximate analysis}

The exact results presented above are cumbersome to calculate and parameter dependency is hard to interpret. In the following, we seek simple approximate but accurate formulas for seating and release configurations, as well as angular position, velocity and acceleration of the driven sprocket.

In obtaining approximate solutions it is utilized that contact angles angles $\theta_{2}$ and $\theta_{4}$ make small variations about $\pi / 2$. This is utilized by introducing shifted angles, marked by a tilde, which are bounded by the angle $\alpha$, c.f. Fig. 2, thus:

$$
\begin{aligned}
& \theta_{2}=\pi / 2+\varepsilon \tilde{\theta}_{2}, \quad \varepsilon \tilde{\theta}_{2} \in\left[-\alpha_{o}, \alpha_{o}\right], \\
& \theta_{4}=\pi / 2+\varepsilon \tilde{\theta}_{4}, \quad \varepsilon \tilde{\theta}_{4} \in\left[-\alpha_{c}, \alpha_{c}\right],
\end{aligned}
$$

where $\varepsilon$ here and below is used to bookmark small terms. It will also be used that span slope $\theta_{3}$ is small, with a conservative estimate for an upper limit given by

$$
\tan \theta_{3} \leq \frac{R_{c}-r_{c}+R_{o}-r_{o}}{x_{c}}
$$


obtained by recognizing that the lower and upper limit of a span endpoints are, respectively, the circumscribed circle radius $r$ and the pitch circle radius $R$. The estimate gives $\theta_{3}<0.015 \mathrm{rad}$ and $\alpha \simeq 0.25$ in the case $n_{o}=n_{c}=12$ and $N=7$. Since most real chain drives have sprockets with more than 12 teeth, and spans more than 8 pitches long, these assumptions are indeed appropriate.

All parameters are nondimensionalized to reduce the number of variables and make the order of magnitude of the various terms easily comparable. All lengths are nondimensionalized by $L$, and nondimensional parameters are identified by over-bars,

$$
\bar{R}_{c}=\frac{R_{c}}{L}, \quad \bar{R}_{o}=\frac{R_{O}}{L}, \quad \bar{r}_{c}=\frac{r_{c}}{L}, \quad \bar{r}_{o}=\frac{r_{o}}{L}, \quad \bar{p}=\frac{p}{L},
$$

from which it follows that $\bar{x}_{c}=1+f \bar{p}$ and $\bar{y}_{c}=\bar{r}_{o}-\bar{r}_{c}$.

\subsection{Approximate seating configuration}

Simple expressions for the configuration where a roller seats on the driver sprocket will be obtained from an approximate solution of (17). Looking at Fig. 2 it is expected that $\theta_{4 s}$ is close to $z_{3}$, so we let

$$
\theta_{4 s}=\frac{\pi}{2}+\alpha_{c}+\varepsilon \hat{\theta}_{4 s}
$$

where the shifted variable is marked with a hat, and $\varepsilon \hat{\theta}_{4 s}$ is assumed to be small. We insert this into (17), expand the trigonometric functions and approximate them by the linear part of their Taylor expansions, e.g. $\sin \left(\pi / 2+\alpha_{c}+\varepsilon \hat{\theta}_{4 s}\right) \approx 1-\alpha_{c} \varepsilon \hat{\theta}_{4 s}$. With this the approximate solution for $\varepsilon \hat{\theta}_{4 s}$ can be expressed as,

$$
\varepsilon \hat{\theta}_{4 s} \approx \frac{B_{s}+C_{s}-A_{s} \alpha_{c}}{A_{s}+B_{s} \alpha_{c}}
$$


We then simplify the coefficients $A_{s}, B_{s}$ and $C_{s}$ from (18) by inserting $\sin \theta_{1}=y_{c} /|O C|, \cos \theta_{1}=x_{c} /|O C|, \gamma=\pi / 2+\alpha_{c}$ and $r_{1}=|O C|$. Next substitute $x_{c}=L+f p, y_{c}=r_{o}-r_{c}$, the lengths given by (13) with $h=0$ and nondimensionalize using (35). Approximating the resulting coefficients is done by assuming the radius of the inscribed- and pitch circles to be identical. This follows from expressing the radii in (1) using only the first term of the Taylor expansions for the trigonometric functions, i.e. $\tan \alpha \approx \sin \alpha \approx \alpha$. Inserting these approximate expression for the radii as well as $\cos \alpha \approx 1$ gives

$$
\begin{aligned}
& \hat{A}_{s}=\bar{p}\left(\frac{\bar{p}}{\alpha_{c}}+2 \alpha_{c}\right) f+\frac{\bar{p}}{\alpha_{o}}+2 \alpha_{c} \\
& \hat{B}_{s}=-2 \bar{p} f+\frac{\bar{p}^{2}}{2}\left(\frac{1}{\alpha_{c} \alpha_{o}}-\frac{1}{\alpha_{c}^{2}}\right)+\bar{p}\left(\frac{\alpha_{c}}{\alpha_{o}}-1\right)-2, \\
& \hat{C}_{s}=\bar{p}^{2} f^{2}+2 \bar{p} f+\frac{\bar{p}^{2}}{2}\left(\frac{1}{\alpha_{c}^{2}}-\frac{1}{\alpha_{c} \alpha_{o}}\right)+2+\bar{p} .
\end{aligned}
$$

These approximations of the coefficients in (18) are marked with a hat and nondimensionalized by $L^{2}$. We then approximate $\varepsilon \hat{\theta}_{4 s}$ by a polynomial in $f$,

$$
\varepsilon \hat{\theta}_{4 s}=a_{0}+a_{1} f+a_{2} f^{2} .
$$

We substitute this and (38) into (37) and determine the coefficients $a_{0}, a_{1}$ and $a_{2}$ by equating to zero like powers of $f$, which gives:

$$
\begin{aligned}
& a_{0}=\frac{-4 \alpha_{c}^{3} \alpha_{o}}{\bar{p}\left(\left(2 \alpha_{c}^{2}+\bar{p}\right)\left(\alpha_{c}-\alpha_{o}\right)+2 \alpha_{c}\right)}, \\
& a_{1}=\frac{-2\left(2 \alpha_{c}^{2}+\bar{p}\right) \alpha_{c} \alpha_{o}}{\left(2 \alpha_{c}^{2}+\bar{p}\right)\left(\alpha_{c}-\alpha_{o}\right)+2 \alpha_{c}}, \\
& a_{2}=\frac{2 \bar{p}\left(\bar{p} \alpha_{o}+\alpha_{c}\right) \alpha_{o}}{\left(2 \alpha_{c}^{2}+\bar{p}\right)\left(\alpha_{c}-\alpha_{o}\right)+2 \alpha_{c}} .
\end{aligned}
$$

These coefficients are approximated by retaining only the dominating linear terms, giving $a_{o} \approx 0, a_{1} \approx-\bar{p} \alpha_{o}$, and $a_{2} \approx \bar{p} \alpha_{o}$. With these coefficients substitute (39) into (36) to get

$$
\theta_{4 s} \approx \frac{\pi}{2}+\alpha_{c}+\bar{p} \alpha_{o} f(f-1)
$$


Using this, the angle of the span when a roller seats on the driver sprocket can be found from (16):

$$
\theta_{3 s} \approx \bar{p} \alpha_{o} f(f-1) .
$$

With real chain drives, both $\bar{p}$ and $\alpha_{o}$ are expected to be small.

A simple expression for $\theta_{2 s}$ is determined using the approximate results for $\theta_{4 s}$ and $\theta_{3 s}$ : In (14)-(15), use that $\theta_{3 s} \approx 0$ and insert $\cos \theta_{3} \approx 1$ and $\sin \theta_{3} \approx 0$. Shift to the variable $\varepsilon \tilde{\theta}_{2 s}$ using (33) and insert the approximation $\theta_{4 s}=\pi / 2+\alpha_{c}$, including only the first term of the Taylor expansions of the trigonometric functions. Divide the two resulting equations to eliminate $r_{2}$ and obtain an equation for $\varepsilon \tilde{\theta}_{2 s}$. Nondimensionalize using (13) and (35) with $h=0$, use $\bar{r}_{c} \approx \bar{R}_{c}$ and $\bar{R}_{c} \approx \bar{p} /\left(2 \alpha_{c}\right)$, shift back the variable to obtain:

$$
\theta_{2 s} \approx \pi / 2+\alpha_{o}(1-2 f) .
$$

\subsection{Approximate release configuration}

To determine simple approximate expressions for the release configuration one can proceed as for the seating configuration. In this case however, shift the variable in (23) by introducing $\theta_{2 r}=\pi / 2-\alpha_{c}-\varepsilon \hat{\theta}_{2 r}$. Following the same principal steps as in section 3.1 leads to

$$
\begin{aligned}
& \theta_{2 r} \approx \pi / 2-\alpha_{o}-\bar{p} \alpha_{c} f(f-1), \\
& \theta_{3 r} \approx-\bar{p} \alpha_{c} f(f-1) \\
& \theta_{4 r} \approx \pi / 2-\alpha_{c}(1-2 f),
\end{aligned}
$$

where (22) has been used to calculate $\theta_{3 r}$.

\subsection{Phase between seating and release}

The excitation of the chain drive coming from the polygonal effect depends on the phase between the seating and release of the rollers. Such a 
phase can be defined in various ways, but one choice would be

$$
\psi=\theta_{4 s}-\theta_{4 r} \approx 2 \alpha_{c}(1-f),
$$

where the approximation have been obtained using (46) and (41), in which the small term $\bar{p} \alpha_{o}$ have been assumed vanishing.

\subsection{Approximate I/O-analysis}

Expressing the driven sprocket angular position directly as a function of the driver position and span length requires an approximate solution of (27). Shifting to $\varepsilon \tilde{\theta}_{2}$ using (33) and approximating the trigonometric functions by the linear terms of their Taylor expansion gives

$$
\varepsilon \tilde{\theta}_{2} \approx \frac{B+C}{A} .
$$

We then simplify the coefficients in (28) using $\sin \theta_{1}=y_{c} /|O C|, \cos \theta_{1}=$ $x_{c} /|O C|$ and (13), substitute $x_{c}=L+f p, y_{c}=r_{o}-r_{c}$, the lengths given by (13) with $h \neq 0$ and nondimensionalize using (35). Shifting $\theta_{2}$ and $\theta_{4}$ according to (33) and approximating the resulting coefficients assuming $R_{o} \approx r_{o}$ and $R_{c} \approx r_{c}$, and using the first term of the Taylor expansions of the trigonometric functions gives

$$
\begin{aligned}
& \tilde{A}=-2 \bar{R}_{o}(1+f \bar{p})-2 \bar{R}_{c} \bar{R}_{o} \varepsilon \tilde{\theta}_{4}, \\
& \tilde{B}=-2 \bar{R}_{o}^{2}, \\
& \tilde{C}=2 R_{o}^{2}+(1+f \bar{p})^{2}-(1+h \bar{p})^{2}-2 R_{c}(1+f \bar{p}) \varepsilon \tilde{\theta}_{4},
\end{aligned}
$$

where the approximate coefficients (marked by a tilde) have been nondimensionalized by $L^{2}$. Inserting (49) into (48) and shifting back the angles with (33) gives

$$
\theta_{2} \approx \frac{\pi}{2}+\frac{\bar{p}(f-h)\left(1+\frac{1}{2} \bar{p}(f+h)\right)-\bar{R}_{c}(1+f \bar{p})\left(\theta_{4}-\pi / 2\right)}{\bar{R}_{o} \bar{R}_{c}\left(\theta_{4}-\pi / 2\right)-\bar{R}_{o}(1+f \bar{p})},
$$

where $h$ varies discontinuously with $\theta_{4}$ according to (26). 


\subsection{Velocity}

The exact driven sprocket angular velocity given by (30) can be approximated. We do this by neglecting $\theta_{3}$ in (30), shifting the angles $\theta_{2}$ and $\theta_{4}$ using (33), shifting to cosine functions and approximating them using the first two terms of their Taylor expansions. Then substituting the nondimensional radii gives

$$
\omega_{2} \approx \omega_{4} \frac{\bar{R}_{c}\left(1-\left(\theta_{4}-\pi / 2\right)^{2}\right)}{\bar{R}_{o}\left(1-\left(\theta_{2}-\pi / 2\right)^{2}\right)} .
$$

Since it is relevant for the approximation of the acceleration, the span velocity $\omega_{3}$ given by (31) is also considered. The span angle $\theta_{3}$ is again assumed vanishing and the trigonometric functions are approximated by the first term of their Taylor expansion, which gives

$$
\omega_{3} \approx \omega_{4} \frac{\bar{R}_{c}}{1+h \bar{p}}\left(\theta_{2}-\theta_{4}\right)
$$

For a conservative estimate of the order of magnitude of $\omega_{3}$ assume $\left|\theta_{2}-\theta_{4}\right| \leq$ 0.5 and $1 / \bar{R}_{c} \approx 5$ to get $\omega_{3} \approx \omega_{4} / 10$. Thus, $\omega_{3}$ is one order of magnitude smaller than $\omega_{4}$ when spans are long compared to the sprocket size $\bar{R}_{c}$.

\subsection{Acceleration}

In the exact expression for the angular acceleration given by (32) we assume both $\theta_{3}$ and $\omega_{3}$ to be vanishing. Including only the first term of the Taylor expansions of the trigonometric functions then gives an approximate expression for the angular acceleration of the driven sprocket:

$$
\alpha_{2} \approx \alpha_{4} \frac{\omega_{2}}{\omega_{4}}+\omega_{2}^{2}\left(\theta_{2}-\pi / 2\right)-\omega_{4}^{2} \frac{R_{c}}{R_{o}}\left(\theta_{4}-\pi / 2\right) .
$$

In the expressions for both angular velocity and acceleration the angles $\theta_{2}$ and $\theta_{4}$ jumps discontinuously every time a roller is released from and seated on the sprockets, respectively. 


\section{Example results}

\subsection{Test configurations}

Three different chain drive configurations are used when presenting results of the kinematic analysis, with properties as listed in Table 1. The

\begin{tabular}{lccc} 
& $n_{c}$ & $n_{o}$ & $N$ \\
\hline Coarse & 6 & 9 & 4 \\
Medium & 12 & 18 & 11 \\
Fine & 21 & 63 & 34
\end{tabular}

Table 1: Configurations

pitch fraction $f=\{0,0.5,0.75\}$ will be stated explicitly as results are presented. These values of $f$ are chosen because minimum and maximum variation of the driven sprocket velocity occurs for $f=0$ and $f=0.5$, respectively, $[4,5,8]$. Most previous studies have utilized either $f=0$ or $f=0.5$, and $f=0.75$ have been chosen to illustrate the general case. The coarse configuration was used in [4] and with a different span length also [8]. Experimental measurements of "angular displacement error" (the difference between angular position and angular position for constant velocity), for the driven sprocket exists for the medium configuration [8]. The fine configuration was also analyzed [10]. A driver angular velocity of $\omega_{4}=100 \mathrm{rpm} \approx 10$ $\mathrm{rad} / \mathrm{s}$ has been used when calculating driven sprocket angular velocity and acceleration.

\subsection{Angular position, velocity and acceleration}

Figure 4 shows the exact and approximate results for the coarse, medium and fine configurations in columns $\mathrm{C}, \mathrm{M}$ and $\mathrm{F}$, respectively. The horizontal 
axis shows the angular position of the driver normalized so that $[0,1]$ correspond to one tooth period, starting when a roller seats on the driver. Exact results were obtained by discretizing $\theta_{4}$ and calculating the configuration of the chain drive for each $f$. The approximate results for driven sprocket angular position, velocity and acceleration are calculated using Equations (50), (51) and (53), respectively, with seating and release positions obtained from the exact analysis.

Angular position of the driven sprocket is shown in Fig. 4(a-b-c). There is seen to be very good agreement between exact and approximate results, especially for the medium and fine configurations which are of most practical importance. The phase $\psi$ between rollers being seated $(\nabla)$ and released $(\triangle)$ is shown with a double arrow in Fig. 4(a). Since the horizontal axis shows one tooth period the phase between seating and release is simply $\psi=1-f$, as shown in Equation (47).

The velocity ratio between the driven and driver sprocket varies during one tooth period and is shown in Fig. 4(d-e-f). All three graphs shows excellent quantitative and qualitative agreement between exact and approximate results. For the coarse, medium and fine configurations the variation of the velocity ratio is seen to be on the first, second and third decimal point, respectively. This demonstrates how the magnitude of the velocity variation decreases rapidly as the number of teeth is increased. The velocity variation was shown empirically to be inversely proportional to $n_{c}^{2}$, assuming $n_{c}<n_{o}$ [8]

In Fig. 4(d-e) one can see the how the velocity ratio variation decreases and smoothens when $f=0$, compared to $f=0.5$. However, as the tooth ratio $n_{c} / n_{o}$ decreases (smaller than about $1 / 3$ ), the effect of changing $f$ becomes less significant. This can be seen in Fig. 4(f), where the curves for $f=0$ 

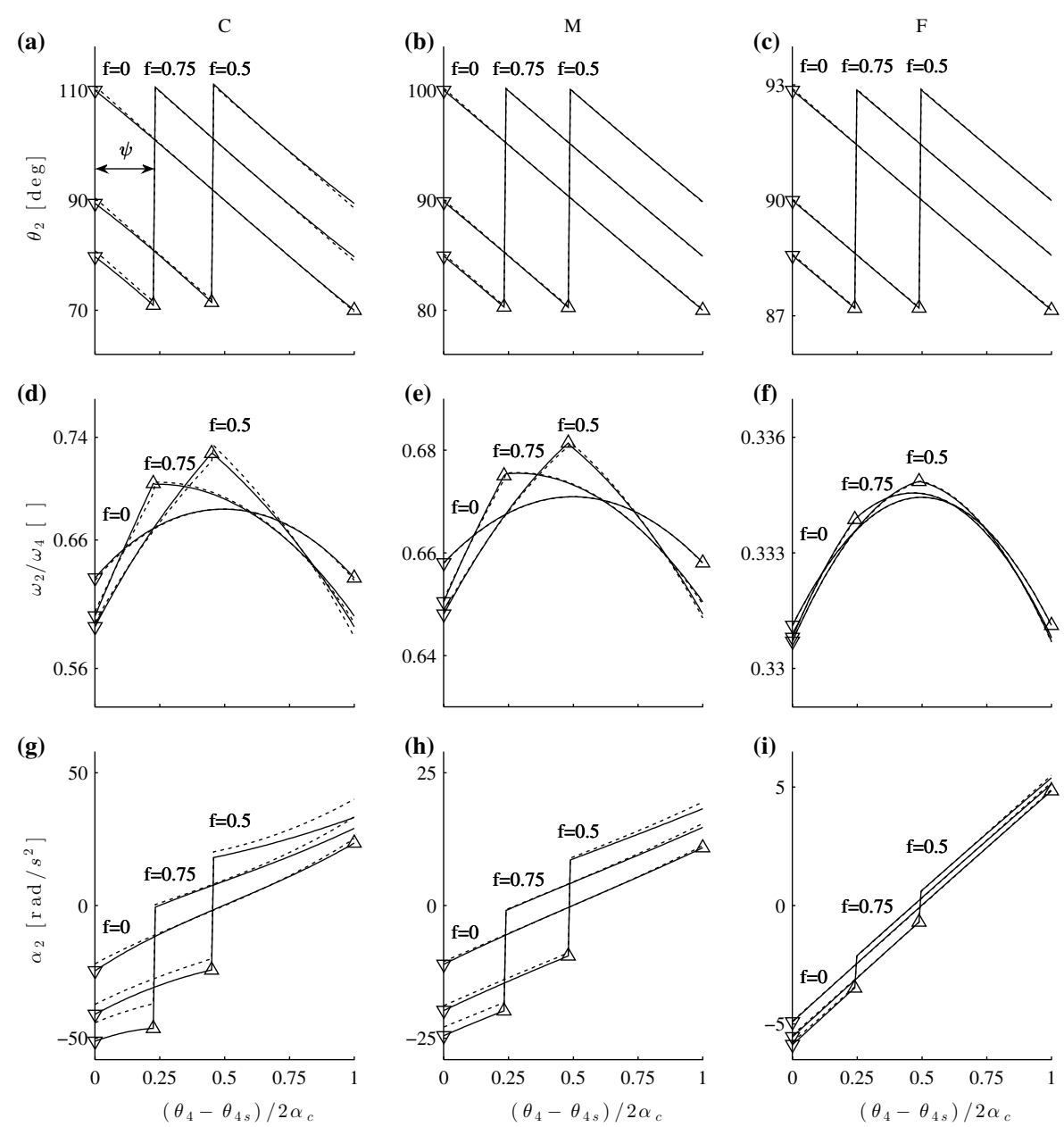

Figure 4: Exact (-) and approximate (--) angular position, velocity and acceleration (first, second and third row, respectively) of the driven sprocket for one tooth period, for the coarse, medium and fine chain drive configuration (first, second and third column, respectively). A roller seating on the driver sprocket is indicated by $\nabla$, and the release of a roller from the driven sprocket is indicated by $\triangle$. 
and $f=0.5$ are practically identical. Lastly, the velocity ratio will only be constant in the special case $n_{c}=n_{o}$ and $f=0$, as can be seen from (51).

The driven sprocket angular acceleration in Figures 4(g-h-i) shows good quantitative and qualitative agreement between exact and approximate results. In the results presented here the driver rotates with constant angular velocity, and despite, that two sudden jumps in angular acceleration occurs when $f \neq 0$, whereas for $f=0$ the acceleration only jumps at the end of the interval. The magnitude of a discontinuous jump occurring when a roller is seated or released decrease rapidly as the number of teeth is increased on the driver- and driven sprocket, respectively. Thus, the discontinuous jumps are most prominent for sprockets with few teeth.

For a driver rotating with constant angular velocity the time between seating and release will be given by $\psi=(1-f) T$, where $T$ is the tooth period. Seating and release will only happen simultaneously if $f=0$. Only in the special case where $f=0$ and $n_{c}=n_{o}$ will the angular velocity and acceleration of the driven- and driver sprockets be equal.

In studies of string and roller chain drive dynamics it is often assumed that polygonal action leads to a parametric excitation described by time harmonic variation of span tension or velocity [17]. However, the driven sprocket acceleration in Fig. 4(g-h-i) is shown to be non-smooth and this could be taken into consideration when modeling chain drive loads.

\subsection{Seating and release angles}

Figure 5 shows the exact angular seating- and release positions for the coarse configuration, together with the closed form approximations. The phase $\psi$ between seating and release given by (47) is indicated with an arrow in Fig. 5(b). In Fig. 5(a) it is seen that the approximation given 
(a)

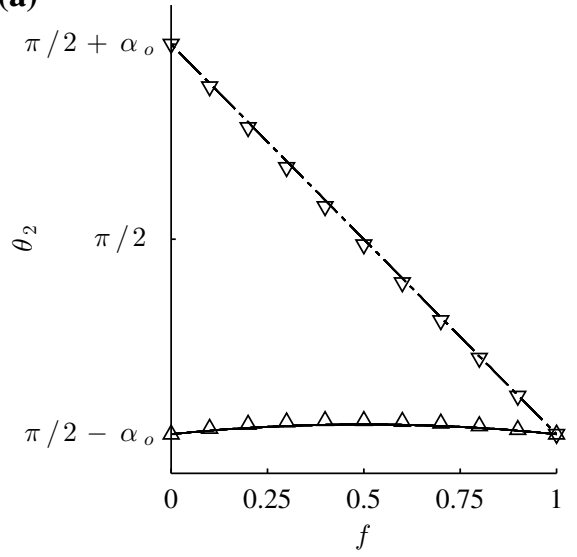

(b)

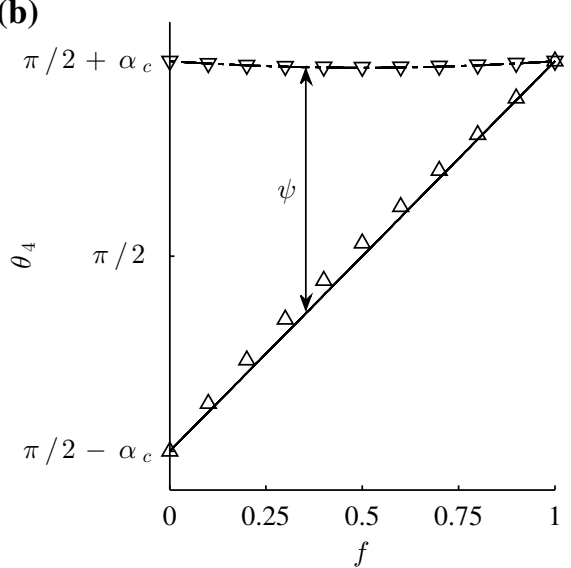

Figure 5: Exact seating $\nabla$ and release angles $\Delta$ as a function of the pitch fraction $f$ for the coarse configuration. Approximations for seating $(-)$ and release $(-\cdot-)$ are shown for (a) the driven sprocket and (b) the driver sprocket.

by (44) capture correctly that $\theta_{2 r}$ is almost constant. There is only slight movement toward the horizontal position $\pi / 2$ for $f$ around 0.5 . This is due to the rise of the span endpoint at the opposite sprocket, e.g. $\theta_{4 r}=\pi / 2$ for $f=0.5$. Similarly for $\theta_{4 s}$ in Fig. $5(\mathrm{~b})$ as predicted by (41).

The driven sprocket angular seating position $\theta_{2 s}$ is proportional to $f$ and moves between the outer limits given by $z_{1,2}=\pi / 2 \pm \alpha_{o}$ in Fig. 2 , as predicted by the approximation in (43). Similarly, the driver sprocket angular position $\theta_{4 r}$ given by (46) moves between limits $z_{3,4}=\pi / 2 \pm \alpha_{c}$, in agreement with the exact results.

Impact has not been the object of investigation of this work, but it has been shown that the seating position $\theta_{4 s}$ is close to point $z_{3}$ in Fig. 2, as is often assumed in studies of impact and noise $[18,19]$. In Fig. 1 the relative velocity between a seating roller and the driver sprocket $\mathbf{u}_{1}-\mathbf{u}_{2}$, is therefore practically independent of $f$. Assuming the span is a straight line, the main 
design factor affecting the relative velocity, besides the angular velocity of the driver, is therefore the number of teeth on the driver sprocket (the pitch angle).

Results are not presented here for the medium and fine configurations, but when calculated the curves for $\theta_{2 r}$ and $\theta_{4 s}$ straighten and approximations improve slightly as the number of teeth increase.

\subsection{Approximation error}

The error is calculated between the approximate and exact results for the driven sprocket angular position, velocity and acceleration. The error is calculated as the normalized root mean square deviation:

$$
\epsilon_{x}=\sqrt{\frac{1}{n} \sum_{i=1}^{n}\left(\tilde{x}_{i}-x_{i}\right)^{2}} / \sqrt{\frac{1}{n} \sum_{i=1}^{n} x_{i}^{2}},
$$

where $\tilde{x}_{i}$ represent the approximation and $x_{i}$ the exact value of sample $i$. Figure 6 shows approximation errors for angular position $\theta_{2}$, velocity $\omega_{2}$ and acceleration $\alpha_{2}$.

The errors in Fig. 6 were calculated using a tooth ratio of unity. Since the trigonometric functions were approximated by Fourier expansions the largest errors are to be expected for the chain drive configurations with the lowest number of teeth, hence $n=n_{o}=n_{c}$. Obtaining the approximate results made use of the span slope being small, so it is expected that the approximations will improve as span length is increased. The errors presented are the maximum errors obtained for $n=10 . .40$, when the span length and pitch fraction are varied as $N=10 \ldots 40$ and $f=0.1 \ldots 0.9$. Error calculations for different tooth ratios are not presented here but show both quantitative and qualitative similarity to Fig. 6 . 
Approximation errors for the driven sprocket angular position $\tilde{\theta}_{2}$ in Fig. 6(a) are less than $0.5 \%$, and decreasing when the chain span becomes longer and the number of teeth on the sprockets increase, as expected. Similarly for the approximation of the driven sprocket angular velocity $\tilde{\omega}_{2}$, where errors are less than $0.1 \%$. The approximation errors for the driven sprocket angular acceleration $\tilde{\alpha}_{2}$ are less than $10 \%$ for $n, N>12$ and reduce to a level of about $5 \%$.

(a)

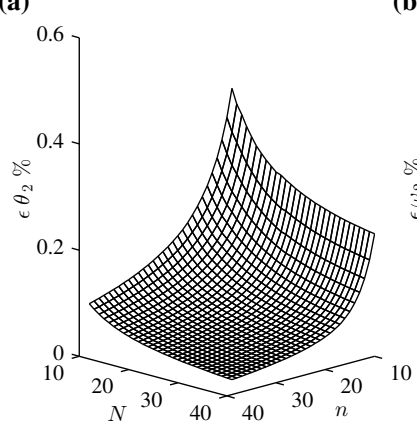

(b)

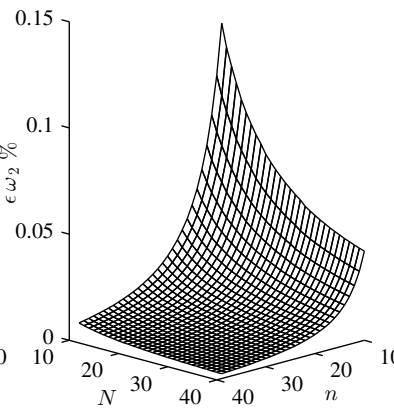

(c)

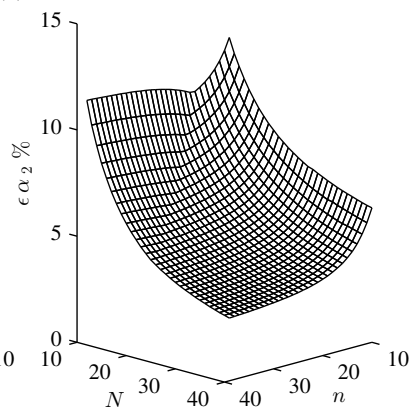

Figure 6: Error between exact and approximate angular (a) position, (b) velocity, (c) acceleration of the driven sprocket.

\section{Comparison with multibody simulation}

The exact kinematic prediction of the chain span motion is compared with results from multibody simulation of a roller chain drive. multibody simulations are carried out using the program described in $[11,12]$. In the multibody model the mass of the chain is lumped at the roller center locations, and springs and viscous dampers with constant stiffness- and damping coefficients model the chain links. Clearances between pin and bushings are neglected as well as rotational inertia of the rollers about their center of gravity. Tooth geometry is Type II per ASA B29.1-1950. 
Simulation is carried out for the medium configuration in Table 1 with a chain consisting of 40 links, $p=0.0254 \mathrm{~m}$, and for the shaft center distance $f=0.43$ is chosen to ensure a positive pretension of both the upper and lower spans connecting the two sprockets. Angular velocity of the driver sprocket is $10 \mathrm{rpm}$ and constant. The main parameters for the simulation are chain link stiffness $0.221 \mathrm{GN} / \mathrm{m}$, link mass $0.066 \mathrm{~kg}$ and link damping $0.01 \mathrm{Ns} / \mathrm{m}$. The radius of the driven sprocket is $0.073 \mathrm{~m}$, mass is $1.68 \mathrm{~kg}$, rotational inertia $0.046 \mathrm{~kg} \mathrm{~m}^{2}$ and the rotational damping coefficient is 0.1 $\mathrm{Nms} / \mathrm{rad}$.

Figure 7 shows the curves traced out by the roller centers for the upper span, as the driver sprocket rotates in clockwise direction. Assuming the span moves as a rigid string supported by moving endpoints, the kinematic analysis gives the presented wavy path of the chain span roller centers. The shape of the wavy path depends on the phase between seating and release, i.e. $f$. In Figure 7 the simulation results shows the chain span endpoints moves as predicted by the kinematic analysis. With the simulation results however, the chain is modeled as a flexible string, meaning that each point corresponding to a chain span roller center not only moves as dictated by the chain span endpoints, but vibrates about that (rigid body mode) position, in particular in the lowest, dominating transverse mode. This appears in Fig. 7 as a high-frequency spatial variation of the simulation results, the average of which is in excellent agreement with the kinematic predictions. An increase of e.g. the pretension will affect the frequency of the transverse vibrations but the resulting strain does not introduce deformations that significantly affect the characteristic motion at the span endpoints kinematic.

All together, the comparison demonstrate how the kinematic analysis can be useful for predicting and understanding e.g. simulation and experimental 
results, even when these include dynamic effects. The results in Figure 7 are comparable to an idealized sketch presented for $f=0$ in a discussion of the path of the chain span in chapter 13 [6], and existing simulation results [13].

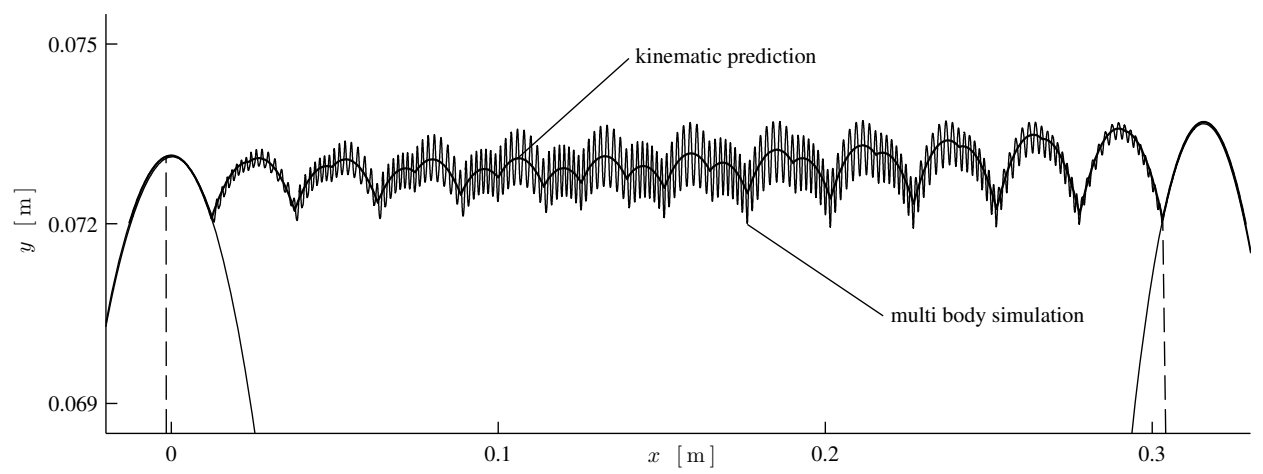

Figure 7: Phase plane for the chain roller centers as predicted by kinematic analysis, and as obtained from multibody simulation. The broken lines -- are drawn from the sprocet centers to the roller centers which are the span endpoints at the instant where a roller seats on the driver sprocket.

Comparison between the kinematic predictions for the angular motion of driven sprocket and multibody simulation results are not presented. In the analysis of multibody simulation results it becomes apparant that the response of the driven sprocket is sensitive to the parameters relating to the dynamics of the sprocket, i.e. inertia, rotational damping and flexibility coming from the connected chain spans. The driven sprocket angular vibration response generally shows resemblence to an impulse respons, where the discontinous excitation results in a smoothend transient respons. An analytical study which includes the coupled dynamics of transverse chain vibration and angular vibration of the driven sprocket is given by Fuglede and Thomsen [20]. 


\section{Conclusion}

A chain drive is modeled as a four-bar mechanism, and equations governing position, velocity and acceleration are presented and solved exactly and approximately. The instantaneous span length is determined, and its discontinuous variation with time is given a simple formulation. It is shown how the chain drive geometry is conveniently nondimensionalized. The seating and release configurations are determined, and simple approximate expressions including only the dominant design parameters are derived.

The driven sprocket angular position, velocity and acceleration is determined, and results presented for a coarse, medium and fine chain drive configurations show very good agreement between exact and approximate results. Errors between exact and approximate results were shown to be small for all practical chain drives. The closed form approximate results provide insight into the effects of changing design parameters, and allows for a convenient estimation of the chain drive kinematics.

An approximate expression for the phase between chain roller seating and release is determined, giving insight into the time intervals between the discontinuous accelerations of the driven sprocket.

Comparison is made between multibody simulation results and analytical kinematic predictions, and there is seen to be very good agreement. It is demonstrated how the kinematic analysis can be used for interpreting e.g. simulation results.

This study treats the case where the span connects the sprockets such that they rotate in the same direction. However, it is expected that the case where the span connects the two sprockets as the inner tangent could be treated following a similar approach. 


\section{References}

[1] K. W. Wang and S. P. Liu. On the noise and vibration of chain drive systems. The Shock and Vibration Digest, 23(4):8-13, 1991.

[2] J. N. Fawcett. Chain and belt drives, a review. The Shock and Vibration Digest, 13(5):5-12, 1981.

[3] Li-Qun Chen. Analysis and control of transverse vibrations of axially moving strings. Applied Mechanics Review, 58(2):91-116, 2005.

[4] G. M. Bartlett. Roller chain drives in theory and practice. Product Engineering, 2(4):253-255, 1931.

[5] R. A. Morrison. Polygonal action in chain drives. Machine Design, 24 (9):155-159, 1952.

[6] R. C. Binder. Mechanics of the Roller Chain Drive: Based on Mathematical Studies by RC Binder. Prentice-Hall, 1956.

[7] S. Mahalingam. Polygonal action in chain drives. Journal of The Franklin Institute, 265(1):23-28, 1958.

[8] G. Bouillon and G. V. Tordion. On polygonal action in roller chain drives. Journal of Engineering for Industry, 87(2):243-250, 1965.

[9] S. R. Turnbull and J. N. Fawcett. An approximate kinematic analysis of the roller chain drive. Proceedings of the Fourth World Congress on Theory of Machines and Mechanisms, pages 907-911, 1975.

[10] C. K. Chen and F. Freudenstein. Toward a more exact kinematics of roller chain drives. Journal of Mechanisms Transmissions and Automation in Design-Transactions of the ASME, 110(3):269-275, 1988. 
[11] S. L. Pedersen, J. M. Hansen, and J. A. C. Ambrosio. A roller chain drive model including contact with guide-bars. Multibody System Dynamics, 12(3):285-301, 2004.

[12] S. L. Pedersen. Model of contact between rollers and sprockets in chaindrive systems. Archive of Applied Mechanics, 74(7):489-508, 2005.

[13] C. M. Pereira, J. A. Ambrosio, and A. L. Ramalho. A methodology for the generation of planar models for multibody chain drives. Multibody System Dynamics, 24(3):303-324, 2010.

[14] J. Ambrósio, C. Pereira, and A. Ramalho. Roller chain drives multibody models using a generalized revolute clearance joint formulation. In Proceedings of the 23rd International Congress of Theoretical and Applied Mechanics, 2012.

[15] M. R. Smith and L. Maunder. Inertia forces in four-bar linkage. Journal of Mechanical Engineering Science, 9(3):218-225, 1967.

[16] K.J. Waldron and G.L. Kinzel. Kinematics, dynamics, and design of machinery. Wiley, 1999.

[17] S. T. Ariaratnam and S. F. Asokanthan. Dynamic stability of chain drives. Journal of Mechanisms Transmissions and Automation in Design - Transactions of the ASME, 109(3):412-418, 1987.

[18] W. Choi and G. E. Johnson. Vibration of roller chain drives at low, medium and high operating speeds. American Society of Mechanical Engineers, Design Engineering Division (Publication) DE, 63:29-40, 1993. 
[19] H. Zheng, Y. Y. Wang, G. R. Liu, K. Y. Lam, K. P. Quek, T. Ito, and Y. Noguchi. Efficient modelling and prediction of meshing noise from chain drives. Journal of Sound and Vibration, 245(1):133-150, 2001.

[20] N. Fuglede and J. J. Thomsen. Kinematic and dynamic modeling and approximate analysis of a roller chain drives. Journal of Sound and Vibration, in print, 2016. 